\title{
18. Kollektivität, Partizipation und Solidarität in einer zeitgemäßen Bioethik
}

\subsection{Einleitung}

Wiederholt hat Bundespräsident Frank-Walter Steinmeier im Kontext der COVID19-Pandemie Solidarität eingefordert: Solidarität unter den Ländern der Europäischen Union in der Zusammenarbeit in Politik, Wirtschaft und Wissenschaft sowie Solidarität in der globalen Verteilung von Impfstoffen gegen das Coronavirus SARS-CoV-2 (Tagesschau, 2020; Tagesschau, 2021). Ähnlich virulent wie der Begriff der Solidarität kursiert seit einiger Zeit die Forderung nach Partizipation von Beteiligten und Betroffenen in der Gesundheitsforschung und -politik, aber auch in medizinethischen Diskussionen (siehe auch Winkler/Prainsack, Kap. 17).

„Öffentliche Beteiligung“ im Zusammenhang mit datenintensiver Forschung in Medizin und Gesundheitswesen boomt, insbesondere wenn es um die Kombination von genomischen und anderen, z. B mithilfe von Smartphones und Fitnessarmbändern („Wearables“) erzeugten, gesundheitsbezogenen Daten geht (Woolley et al., 2016). Diese Entwicklung weckt große Hoffnungen auf mehr personalisierte Behandlungen. Die Zusammenführung großer Datensätze aus unterschiedlichen Quellen soll die Krankheitsvorhersage sowie Behandlungsentscheidungen durch die Stratifizierung von Patient*innen nach bestimmten Krankheitsuntergruppen erleichtern. Die Teilnahme der Patient*innen an datenintensiver medizinischer Forschung und Behandlung verweist dabei in mehrfacher Hinsicht auf Kollektivität. Zum einen werden methodisch gesehen häufig bestimmte Patient*innengruppen angesprochen, ihre Datensätze der Forschung zur Verfügung zu stellen, zum anderen werden in der stratifizierten Auswertung zukünftige, neue Patient*innen-Kollektive klassifiziert, z. B. eine Hochrisikogruppe für Demenzerkrankungen oder onkologische Patient*innen, die bestimmte Medikamente nicht vertragen. Auch das Konzept der Solidarität bezieht sich auf Kollektivität (siehe unten). Beide Konzepte, Beteiligung und Solidarität, deuten in ihrer Funktion auf eine 
Art „sozialen Kitt“ hin: Sie sind relationale Konzepte, die soziale Bindung und Bezüglichkeiten zu einem Kollektiv in ihrer Theoretisierung berücksichtigen.

Im Bereich der datenintensiven Medizinforschung werden Partizipation und Solidarität mittlerweile eng aufeinander bezogen, denn die patientenseitige Teilnahme und Beteiligung wird mit dem Verweis auf Solidarität motiviert (vgl. Neuhaus, 2020; siehe Winkler/Prainsack, Kap. 17). Partizipation und Solidarität sind inzwischen Gegenstand von sozial-, kulturwissenschaftlichen und bioethischen Diskursen. Die gemeinsame theoretische Schnittstelle, das Kollektive, bleibt jedoch meist unterbestimmt.

Unsere Alltagssprache nimmt die Existenz und Relevanz von Kollektiven als selbstverständlich hin. Häufig berufen wir uns auf kollektive Entitäten wie Nationen, die Öffentlichkeit, die Ärzteschaft oder die Familie. Doch was zeichnet Kollektive eigentlich aus und welches Verständnis ist für eine zeitgemäße Bioethik sinnvoll? Die aktuellen ethischen und sozialen Herausforderungen im Umgang mit der COVID-19-Pandemie machen die Notwendigkeit augenscheinlich, sich mit Kollektivität intensiver auseinanderzusetzen. In der bioethischen Theorie fehlen indes noch weitgehend Ansätze, diese Dimension systematisch und differenziert zu bearbeiten. Ein differenziertes Verständnis von Kollektivität bildet zudem eine zentrale Voraussetzung für eben jene ideengeschichtlich und normativ gehaltvollen Konzepte, die seit einiger Zeit vermehrt in öffentlichen und politischen Diskussionen und im bioethischen Diskurs aufgerufen werden: Partizipation und Solidarität. Im Folgenden wollen wir daher einen kurzen Überblick über diese drei Konzepte geben.

\subsection{Wann ist Kollektivität normativ relevant?}

Ist ein Kollektiv nur eine Ansammlung von Individuen oder hat es eine über seine Mitglieder hinausgehende Existenzform? Nach dem Prinzip der kollektiven Akteurschaft (engl. „collective agency“) (McKenna, 2006: 19) zeichnen sich normativ bedeutsame Kollektive durch folgende drei Elemente aus: kollektive Identität, kollektive Willensbildung und kollektive Handlung. Nicht alle Kollektive erfüllen diese drei Elemente ausreichend und entsprechend ist eine Differenzierung notwendig.

Der „kollektivistische“ Grundgedanke in der Sozialphilosophie besagt, dass Kollektive einen von ihren Mitgliedern unabhängigen ontologischen Status haben, ein Eigenleben. John Searle hat prominent argumentiert, dass die ersten Formen der menschlichen Zivilisation aufgrund von Biologie und Funktion der Sprache kollektiv waren (Searle, 1990 und 2010). Individuelle Absichten lassen sich von gemeinsamen Absichten unterscheiden und wir können klar zwischen „Ich“ und „Wir“ unterscheiden. Kollektives Handeln zeichnet sich hingegen nach Bratman (1993 und 2006) dadurch aus, dass 
eine geteilte Absicht zu einer gemeinsamen Handlung führt, wenn zwei oder mehrere Personen die gleiche Absicht zeigen, etwas gemeinsam zu tun, wenn sie Wissen über diese gemeinsame Absicht haben und schließlich bereit sind, nach dieser Absicht zu handeln, einschließlich vorbereitender Handlungen. Dieses Verständnis von kollektivem Handeln ist für viele politisch-ethische Überlegungen zentral, z. B. wenn es um Formen der gesellschaftlichen Beteiligung und Teilhabe geht. Daran schließt eine dritte, auch für die Bioethik relevante Fragestellung an: Wen stellen wir in das Zentrum unserer politisch-ethischen Überlegungen - das Individuum oder das Kollektiv? Oder andersherum formuliert, wer ist es wert, im Diskurs „vertreten“ zu werden - das Individuum oder das Kollektiv? Gerade in der Diskussion um ethisch angemessene Maßnahmen zur Eindämmung der COVID-19-Pandemie ist dieser Konflikt zwischen Individual- oder Kollektivinteressen vielerorts sehr sichtbar aufgetreten - und sowohl ethische als auch politische Lager sind hier oft gespalten. Was sich bereits im Zuge normativer Reflexion auf datenintensive Medizinforschung ${ }^{1}$ (z. B. Genomforschung, Erforschung von kardiovaskulären Erkrankungen, Diabetes und psychischen Krankheiten) in den letzten Jahren abzeichnete, wurde durch die Situation um Corona überdeutlich: die ausschließliche Perspektive auf Individuen, wie sie die liberale, analytische Medizinethik lange dominierte, ist verengend. Sie ist ungenügend, um die aktuellen Herausforderungen in der Gesundheitsversorgung und der Gesundheits- bzw. Forschungspolitik reflexiv und konzeptuell einzuholen. Die angemessenere Antwort ist, dass wir in unseren zukünftigen Ansätzen immer beide Dimensionen, das Individuelle und das Kollektive, sowie deren Beziehung im Blick haben sollten.

In diesem Sinne sind auch Gemeinschaft und Kollektiv untrennbar mit unserer Wahrnehmung der persönlichen Identität und persönlicher Rechte verbunden (vgl. Kymlicka, 1991; Taylor, 1995; Young, 1997), weil sie immer Teil unserer moralischen Anliegen und Interessen sind. Gemäß diesem Verständnis sorgen wir nicht nur für uns selbst, sondern immer auch für die Interessen und Rechte derer, die wir lieben oder um die wir uns sorgen. Dazu gehören Familien, Freunde und in manchen Fällen auch weitere Gemeinschaften. Wir brauchen daher ein differenziertes Bild von der Verschiedenartigkeit von Kollektiven, um ihre jeweiligen ontologischen und normativen Grundan-

1 Einige sprechen auch von „Big Data“-basierter Forschung bzw. von „biomedizinischen Big Data“ (vgl. z. B. Mittelstadt/Floridi, 2016), wir bevorzugen den Terminus der „datenintensiven Medizin“, da er den kontroversen Begriff „Big Data“ umgeht. Unter „datenintensive Medizin“ fassen wir sämtliche medizinische Praxis und Forschung, die mit großen Datenmengen operiert, z. B. Forschung mit Genomdaten oder auch Daten aus stationären und mobilen Messgeräten. Damit verbunden ist die Anwendung von unterschiedlichen Computertechnologien und methodischen Zugängen in der Datenanalyse und auswertung, wie z. B. Machine Learning und Künstliche Intelligenz. 
nahmen zu beleuchten. Kollektive von bioethischer Relevanz sind nicht von spontaner, kurzlebiger Natur, sondern zeichnen sich eher durch interne Struktur und eine gewisse Kontinuität aus. ${ }^{2}$ Eine Grundtypologie unterscheidet zwischen freiwilligen, unfreiwilligen und affirmativen Kollektiven (Schicktanz, in Vorbereitung). Freiwillige Kollektive bestehen aus einzelnen Mitgliedern, die sich ohne Zwang als einer Gruppe zugehörig klassifizieren. Außerdem ist die „Wir-Identität“ der Gruppe ein Ziel an sich und nicht nur instrumentell gemeint. Gemeinsames Handeln basiert hier auf der Kooperation zur Planung und Durchführung einer sozialen oder auch politischen Aktion. Beispiele für freiwillige Kollektive finden sich in politischen Parteien, Freizeitvereinen und Berufsverbänden sowie in einigen Formen von Wirtschaftsverbänden (z. B. „Farmerkollekti$\left.v^{(\prime)}\right)$. Beitritt und Austritt sind freie Entscheidungen ihrer Mitglieder und folgen nicht autoritativen Anweisungen. Im Kontext der Bioethik und der Politik des Gesundheitswesens stellen Berufs- oder Wirtschaftsverbände solche freiwilligen Gruppen dar.

Am anderen Ende des Spektrums klassifizieren wir unfreiwillige Kollektive. Mitglieder solcher Kollektive werden nicht durch ihre eigene Wahl zu Mitgliedern, sondern durch kategorische, externe Zuschreibung in einem Prozess des „Othering“ (Spivak, 1985; Johnson et al., 2004). Sie geht oft Hand in Hand mit sozialer Distanzierung und formalen Sozialkategorien. Typische Beispiele sind Einordnungen entlang von „Rasse“, Geschlecht, Ethnie, Kaste, Klasse, Schichtzugehörigkeit oder Behinderung. Othering verstärkt oft essentialistische und manchmal sogar biologistische Vorstellungen von der Identität einer Person und kann schließlich auch zu Stigmatisierung und Diskriminierung führen. Gerade im Bereich der Medizin werden zuweilen solche sozialen $\mathrm{Zu}-$ schreibungen wie Behinderung, Geschlecht oder Ethnie vorgenommen, ohne sich zu vergewissern, ob die Betroffenen sich selbst dem zugehörig fühlen. Besteht hier eine Kluft zwischen Selbst- und Fremdklassifizierung, ist dies ethisch äußerst problematisch.

Der dritte Typ ist das affirmative Kollektiv, welches für die Bioethik sehr bedeutend ist. Es kann als Untertyp der unfreiwilligen Kollektivität verstanden werden. In einem wesentlichen Unterschied dazu jedoch akzeptieren und bejahen Mitglieder affirmativer Kollektive die von außen zugeschriebene soziale Kategorisierung und eignen sie sich an, wobei sie sie auch positiv aufwerten. Sie nutzen diese gar zur sozialen oder politischen Mobilisierung, z. B. um gegen soziale Ausgrenzung vorzugehen. Auf die externe Kategorisierung folgt also in einem anschließenden, bewussten Akt eine Selbstkategorisierung und aktive Mitgliedschaft. Ein historisches Beispiel hierfür sind die Frauen-

2 Aus soziologischer Perspektive lässt sich der Begriff des „Kollektivs“ als Oberbegriff für sämtliche Formen überindividueller Aggregation, Gruppierung und Verbundenheit verstehen (vgl. Rehbein, 2015; Marschelke, 2019). 
rechts- oder Behindertenbewegungen (Williams, 1998). Dieser dritte Typ umfasst für die bioethische Diskussion relevante Gruppen wie Patient*innen- und Behindertenverbände oder die LGBTQ-Community, aber auch die Familie (Beier et al., 2016; Jongsma et al., 2018). Diese Kollektive sind in sich nicht per se als harmonisch, homogen zu denken, sondern aufgrund von sozialer Differenz, Intersektionalität und Persönlichkeiten heterogen. Erst durch kollektive Verständigung sind solche Kollektive in der Lage, sich (zumindest kontext- oder zeitabhängig) auf gemeinsame Ziele zu verständigen. Die Familie als Kollektiv erfüllt zwar das Kriterium der unfreiwilligen, aber nachgängigen Affirmation, zeichnet sich jedoch auch durch besondere Vertrauens- und Emotionskomponenten aus (Beier et al., 2016). Dennoch ermöglicht das hier vorgelegte Kollektivkonzept ein modernes Familienverständnis, welches abseits von biologistischen und genealogischen Zuordnungen einerseits und romantisierten oder ideologisch aufgeladenen Annahmen bzgl. Liebe oder Verpflichtung andererseits anzusiedeln ist.

Kollektive Perspektiven sind offensichtlich dann relevant, wenn Entscheidungen von mehr als einem Individuum getroffen werden. Partizipative Entscheidungstheorien gehen grundlegend davon aus, dass Mitglieder eines oder mehrerer Kollektive zusammenkommen, um eine gemeinsame Entscheidung zu treffen.

\subsubsection{Partizipation und Deliberation}

Die Hochkonjunktur von Öffentlichkeits- und Patient*innenbeteiligung verspricht im Kern eine neue Rolle für die Proband*innen: Sie werden als Partner*innen in der Forschung angesprochen, oder als Bürgerforscher*innen (Kaye et al., 2012). Die Bedeutung und die praktischen Implikationen eines solchen Engagements sind jedoch alles andere als klar. Partizipation wurde als ein vages Konzept charakterisiert (Kelty et al., 2015); die Vielfalt der Partizipationsverständnisse, wie sie im Kontext der biomedizinischen Forschung verwendet werden, verstärkt diese Verwirrung (Beier et al., 2019). In Anlehnung an die Bedeutung von Partizipation in der politischen Theorie und der Bioethik schlagen wir drei Bedingungen vor, die Partizipation konstituieren und definieren: die Zustimmung zur individuellen Bereitstellung von Daten oder Biomaterial als notwendige Bedingung und als zwei hinreichende Bedingungen die Konsultation bezüglich forschungs- und gesundheitspolitischer Ziele sowie die Mitarbeit in Entscheidungsprozessen oder sogar als Mitforschende. Dabei ändern und erweitern sich offensichtlich die Rollen: Im ersten Fall als Spender*in, im zweiten als Beratende und im dritten Fall als Mitentscheidende.

Während Beteiligung unterschiedlichste Formate annimmt (z. B. Stakeholder-Gremien, Bürger*innen-Konsensus-Konferenzen, Szenario-Verfahren oder Anhörungen und 
Eingaben mit Betroffenen), besteht breiter Konsens darüber, dass jegliches solcher Verfahren eine gewisse Machtverteilung beinhalten muss: Diejenigen, die von einer Entscheidung betroffen sind, sollen auch einen Einfluss auf den jeweiligen Entscheidungsprozess haben. Dahinter steht das ursprüngliche politische Ideal der Autonomie im Sinne der Beteiligung an der kollektiven Selbstverwaltung. Politische Partizipation ist daher nicht gleichzusetzen mit bloßen Kommunikationsstrategien (z. B. der Staat informiert die Bürger*innen über politische Strategien oder Ziele), sondern muss den Teilnehmenden einen realen Einfluss gewähren (siehe Winkler/Prainsack, Kap. 17). Werden Gruppen von Bürger*innen oder Stakeholder*innen involviert, die zuvor kaum involviert waren, kann dies als Demokratisierung oder als Empowerment verstanden werden, je nach theoretischem Blickwinkel. Weitere ethische Gründe für die Einbeziehung von Laienperspektiven und -moral in gesellschaftliche Debatten über Biowissenschaften und Medizin sind, wie im Kontext der empirisch informierten Bioethik, die Sicherstellung einer angemessenen Berücksichtigung der epistemischen und erfahrungsbezogenen Perspektiven von Patient*innen und anderen Betroffenen. In gewisser Hinsicht können daher auch sozialempirische Erhebungen eine wichtige Rolle für den Ausbau von Beteiligungsformaten spielen, z. B. wenn durch Umfragen oder Interviewstudien wichtige Einsichten von Bürger*innen oder Betroffenen generiert werden, die dann in weiteren Entscheidungsprozessen konkrete Berücksichtigung finden (Schicktanz et al., 2012; Blasimme/Vayena, 2020).

Die häufigen Appelle an Engagement und Partizipation in der datenintensiven Forschung übersehen zuweilen diese normativen Grundlagen der Partizipation (Blasimme/Vayena, 2016). Die (positive) Zukunft von Bioethik und Good Governance (,gutem Steuerungshandeln“) liegt darin, verschiedene Ebenen der Partizipation und ihre demokratietheoretische Verankerung aus normativer Sicht in Erinnerung zu rufen und umzusetzen. Die drei Bedingungen von demokratietheoretisch gehaltvoller Partizipation lassen sich auch als drei fundamentale Ebenen der Partizipation verstehen, die sich in ihrem normativen Anspruch steigern: Wenn die erste, einfachste Ebene der Partizipation als erfüllt gelten soll, sollten Patient*innen ein Recht auf individuelle Einwilligung haben (z. B. auf das Erheben von gesundheitsbezogenen Daten durch die Nutzung von Wearables, Sensoren oder Gesundheits-Apps oder durch das einfache Ausfüllen eines Fragebogens auf einem Tablet-Computer). Initiativen, die implizit, intransparent oder gar unfreiwillig Daten sammeln, können nicht für sich in Anspruch nehmen, die Patient*innen an ihrer Forschung „teilhaben“ zu lassen - vielmehr reduzieren sie die Patient*innen auf Bio-Daten- oder Bio-Material-Liefernde bzw. machen sie zu Objekten statt zu Akteur*innen. Auf der höchsten Ebene der normativen Partizipation wird die Einbeziehung von Lai*innen in allen Phasen des Forschungsprozesses gefordert. Dies ist in der datenintensiven Forschung noch eine Seltenheit. Eine Ausnahme 
ist die US-amerikanische Plattform Patients.Like.Me, die nach den Daten von Patient*innen fragt und aus ihnen eigene Forschungskohorten zusammenstellt (Wicks et al., 2011; siehe auch Winkler/Prainsack, Kap. 17). Allerdings stellen solche Datenkooperationen hohe Anforderungen an die Teilnehmenden, da sie Fähigkeiten (sozial, pädagogisch) und Ressourcen (z. B. Zeit) erfordern (Blasimme/Vayena, 2016). Die Förderung der digitalen Gesundheitskompetenz in der breiten Öffentlichkeit kann ein Schritt sein, um beteiligungsrelevante Fähigkeiten auszubilden (Samerski/Müller, 2019).

Folgende grundlegenden Kriterien sind aus unserer Sicht zu erfüllen, um Beteiligung und Engagement von Patient*innen und Öffentlichkeit in der Zukunft der vielfältigen Lebenswissenschaften- und datenintensiven Forschung zu verbessern (Beier et al., 2019):

a. Projekte sollten die zugrundeliegende Motivation für die Inanspruchnahme des Partizipationsgedankens kritisch reflektieren. Insbesondere ist $\mathrm{zu}$ unterscheiden zwischen rein instrumentellen Nutzungen, um z. B. das Datenvolumen zu erhöhen oder finanzielle Gewinne zu erzielen, und genuin normativen Gründen für die Einbeziehung von Patient*innen, um Forschungsziele an die Bedürfnisse, Präferenzen und Meinungen der Patient*innen anzupassen.

b. Es ist wichtig, transparenter zu machen, ob Patient*innen zu ihrem eigenen medizinischen Nutzen teilnehmen oder ob partizipative Vorstellungen im Sinne eines öffentlichen Engagements, das über individuelle Zwecke hinausgeht, evoziert werden (Warsh, 2014). Folglich ist nicht nur das sog. therapeutische Missverständnis zu vermeiden, sondern auch ein ,partizipatorisches Missverständnis“. Das therapeutische Missverständnis ist ein empirisch gut belegtes Phänomen, bei dem Patient*innen angeben, sich an Forschung zu beteiligen, weil sie davon ausgehen, dass es ihre eigene, aktuelle Therapie positiv beeinflussen wird - obwohl dies nicht der Fall sein kann. Bestimmte rhetorische Figuren in der Aufklärung durch Forschende können das therapeutische Missverständnis befördern (Wöhlke et al., 2018). Ein partizipatorisches Missverständnis läge entsprechend vor, wenn Forschungsteilnehmende glauben - z. B. durch die Art wie sie angesprochen werden -, sie könnten als Beratende oder Mitentscheidende bei Forschungsprozessen auftreten, obwohl letztlich nur ihre Datenspende ersucht wird.

c. Man sollte der Tendenz widerstehen, den Begriff der Beteiligung zu entpolitisieren, indem man den Patient*innen echten Einfluss auf die Forschung abspricht - oder sogar ihr Engagement ausnutzt, um Forschungsarbeit auszulagern oder Zeit und Geld zu sparen (Prainsack, 2017a; Winkler/Prainsack, Kap. 17).

d. Wenn von Patient*innen erwartet wird, dass sie sich an der Governance von Forschung beteiligen, sollten Forschungsprojekte darauf vorbereitet sein, ausreichende 
finanzielle und strukturelle Ressourcen vorzuhalten, damit Patient*innen, Lai*innen oder Patient*innenvertreter*innen effektiv kommunizieren und ihre Meinung äußern können. Letztlich wäre ein gleichberechtigter Zugang zu Finanzmitteln und relevanten Quellen der Wissensproduktion eine notwendige Voraussetzung für ein echtes Machtgleichgewicht und einen gleichberechtigten Status.

e. Weiterhin ist zu bedenken, dass auf kollektiver Ebene die öffentliche Äußerung von grundsätzlichen Bedenken oder gar Ablehnung gegenüber speziellen technologischen Entwicklungen eventuell nur außerhalb der jeweiligen Initiativen möglich ist. Daher müssen gesellschaftliche Debatten jenseits konkreter Partizipationsformate immer noch als wichtiger Teil moderner, demokratischer Gesellschaften angesehen werden. Die gesamtgesellschaftlichen Diskussionen über technologische Entwicklungen sollten dabei genauso wie konkrete bereichsspezifische normative Reflexionen, aber auch die Verzahnung beider Diskussionsebenen, institutionell gestärkt werden, um politischen Einfluss zu erzielen.

\subsubsection{Solidarität}

Aufrufe zur Teilnahme an datenintensiver Forschung werden zunehmend mit einem Appell an Solidarität unterlegt (vgl. Neuhaus, 2020). Dies ist auch im Bereich von Public-Health-Maßnahmen zu beobachten, so aktuell zur Eindämmung und Erforschung des Coronavirus Sars-CoV-2. Generell lässt sich im bioethischen Fachdiskurs seit rund zwei Jahrzenten ein intensiviertes Bemühen um eine bioethische und Governanceorientierte Konzeptualisierung von Solidarität verzeichnen (vgl. z. B. Ter Meulen et al., 2001; Prainsack/Buyx, 2011 und 2016; Woods, 2016; Prainsack, 2017b; Jennings, 2018; Kolers, 2021; siehe Winkler/Prainsack, Kap. 17). Dabei fällt auf, dass der Begriff der Solidarität fachspezifisch und in anglo-amerikanischen, französisch- und deutschsprachigen Kontexten äußerst heterogen verstanden und verwendet wird. Eine von Barbara Prainsack und Alena Buyx im Auftrag des britischen Nuffield Council on Bioethics ausgearbeitete Studie bietet sowohl einen ausführlichen Überblick über die impliziten und expliziten Erwähnungen des Konzepts der Solidarität in der bioethischen und sozialwissenschaftlichen Literatur als auch einen hilfreichen Ausgangspunkt für weitere Diskussionen (Prainsack/Buyx, 2011, in Deutsch 2016 erschienen). Aufbauend auf die Literatursichtung haben sie eine Arbeitsdefinition von Solidarität vorgeschlagen, die laut den Autor*innen eine Art „kleinsten gemeinsamen Nenner“ darstellt (Prainsack/ Buyx, 2016: 82). Solidarität wäre ihnen zufolge als Bündel von Praktiken zu verstehen, „welche die Bereitschaft widerspiegeln, Kosten in Kauf zu nehmen, um anderen zu helfen" (ebd., Herv. i. O.). Dabei basiere die Bereitschaft der Individuen, Kosten zu übernehmen ,auf 
dem Erkennen von Gemeinsamkeiten mit einer anderen Person, oder anderen Personen, in mindestens einer im gegebenen praktischen Kontext relevanten Hinsicht" (ebd., Herv. i. O.). In der Praxis unterscheiden die Autor*innen je nach der Wirkungsbreite und dem Grad der Institutionalisierung drei Ebenen von Solidarität: die interpersonale, die kollektive und die rechtliche Ebene (ebd.: 83, vgl. 83-92). Für die interpersonale Solidarität stellt das Erkennen von Gemeinsamkeiten mit einer anderen Person (oder mehreren anderen Personen) kontextspezifisch eine notwendige Bedingung dar (ebd.: 84). Die Manifestation in „äußerlich erkennbare Praktiken“ (ebd.: 85, Herv. i. O.) bildet das hinreichende Zeichen für das tatsächliche Vorliegen der Praxisform der Solidarität. Verfestigt sich die sozial motivierte interpersonale Solidarität zu einer kollektiven Bereitschaft, so werde kollektive Solidarität praktiziert (ebd.: 87 f.). Als Beispiele hierfür führen Prainsack und Buyx Selbsthilfegruppen, aber auch Krankenversorgungs- und Versicherungssysteme an. Wenn die in der kollektiven Solidarität eingeübten sozialen und moralischen Normen rechtliche und vertragliche Regelungen auf der Ebene des Staates fundieren, könne man schließlich vom höchsten Institutionalisierungsgrad solidarischer Praktiken sprechen.

Dieser Ansatz zur Definition von Solidarität baut auf bestimmten Vorannahmen auf: Zum einen handelt es sich um ein praxeologisches Verständnis, was bedeutet, dass Solidarität weder ein Prinzip noch eine rein regulative Idee oder Tugend ist, sondern sich vielmehr erst im konkreten zwischenmenschlichen Vollzug realisiert und zeigt. Zudem spielen gewisse konsequentialistische Annahmen hinein, z. B. das Inkaufnehmen von Kosten. Solidarische Praktiken basieren gemäß der Arbeitsdefinition von Prainsack und Buyx außerdem auf einem kognitiv-rationalen Erkennen von Gemeinsamkeiten und Ähnlichkeiten zu anderen Menschen oder Kollektiven. Hingegen bleiben emotionale, historische und biografische Bezüge unberücksichtigt. Schließlich wird davon ausgegangen, dass sich Solidarität in unterschiedlichen Verfestigungsgraden und Institutionalisierungsformen empirisch beschreiben lässt.

Ein großer Vorzug der Arbeitsdefinition liegt in der Kombination verschiedener Facetten von Konzeptualisierungen von Solidarität, welche sowohl individuelle als auch relationale Bezüge herausarbeitet. Solidarität wird weder als per se moralisches noch politisches Ideal verstanden. Dies macht diesen Ansatz für die aktuelle biomedizinische Praxis und klassische bioethische, auf individuelle Interessen ausgerichtete, Argumentation anschlussfähig. Allerdings muss man bedenken, dass durch die deskriptiv akzentuierte, empirische Zugangsweise mögliche sozialtheoretische, politisch-theoretische und ideengeschichtliche Dimensionen von Solidarität ausgeblendet werden. Ähnlich wie bei der rhetorischen Verwendung von Partizipation kann das demokratietheoretische und gesellschaftskritische Potenzial von Solidaritätsforderungen über- 
sehen werden (siehe Winkler/Prainsack, Kap. 17). So stellt sich die Frage, wie über eine deskriptive Verwendung hinaus normativ erfasst werden kann, wenn bestimmte Verhaltensweisen als „unsolidarisch“ bezeichnet und somit unmoralisches Verhalten kritisiert wird. Inwiefern ermöglicht die Praxis von Solidaritätszuschreibungen es also, derartige Kritik auf normativ-theoretische Füße zu stellen (vgl. Stjernø, 2009)? Es könnte zudem die Gefahr entstehen, dass politisches und administratives Steuerungshandeln formal im Namen von Solidarität betrieben wird, sich dabei aber der demokratischen Kontrolle und Deliberation entzieht. So können neue Verfahrensweisen, die sich aus überwiegend administrativen Gründen oder Forschungsinteressen als vorteilhaft erweisen - wie beispielsweise die breite Einwilligung (Broad Consent) in die Nutzung von Biomaterial und Gesundheitsdaten - im Namen der Solidarität zur Routine werden, ohne dass deren ethische und politische Implikationen mit Betroffenen diskutiert werden (vgl. Woods, 2016: 222 ff.). Zudem muss schließlich genauer geklärt werden, an welche Kollektive sich Solidaritätsaufforderungen richten sollen. Gerade in der COVID19-Pandemie wurde deutlich, dass gesellschaftliche Maßnahmen zur Eindämmung (wie das Maskentragen, soziale Isolation oder Reisebeschränkungen) unterschiedliche soziale Schichten und Gruppen unterschiedlich hart treffen und sogar bestehende soziale Ungleichheiten vertiefen können (z. B. Allmendinger, 2020; Wachtler/Hoebel, 2020). Hierbei müssen verschiedene Solidaritätsansprüche gegeneinander abgewogen werden. Wie kann Solidarität mit sozial schwachen Gruppen, denen die ökonomischen Folgen bestimmter Einschränkungen besonders zu schaffen machen, obgleich sie gerade in den „systemrelevanten“ Tätigkeiten einen unverzichtbaren Beitrag zur Aufrechterhaltung des gesellschaftlichen Lebens beitragen, ausgeglichen werden mit der Solidarität mit Gruppen, die gesundheitlich besonders gefährdet sind? Welche Gruppen erhalten mehr kollektive Solidarität und wer entscheidet dies im Konfliktfall? Wer darf legitimerweise zu Solidarität aufrufen? Lässt sich gesamtgesellschaftliche Solidarität überhaupt einfordern? Inwiefern stehen sich Datensicherheitsrisiken und Solidaritätsappelle - sowohl in der datenintensiven Medizinforschung als auch in Monitoringmaßnahmen (z. B. durch sog. Pandemie-Apps) auf Bevölkerungsebene - gegenüber? Um diese komplexen Fragen aus bioethischer Sicht zu klären, müssen praktische Definitionen von Solidarität weiter ausbuchstabiert werden. Zugleich setzt hier ergänzend die normative Bedeutung von Kollektivität an - welche Kollektive sind freiwillig, welche unfreiwillig und wie wirkt sich das auf die praktizierte Solidarität aus? Inwiefern müssen Solidaritätspraktiken in einer kollektiven, gesellschaftlichen Auseinandersetzung immer wieder neu verhandelt werden und wer initiiert diese Auseinandersetzung? 


\subsection{Schlussüberlegungen und Ausblick}

Wenn es um datenintensive Forschung geht, stellt sich also nicht nur die Frage, ob sie empirisch funktioniert - die Evidenz hierfür scheint zumindest aussichtsreich (Xu et al., 2019) -, sondern es muss auch genauer normativ geprüft und reflektiert werden, ob die Appelle an Partizipation und Solidarität wirklich in dem jeweiligen Kontext stimmig sind. Teilnahme im Rahmen einer Datenspende für die Forschung kann als solidarischer Akt zu anderen Patient*innen oder zukünftigen Kranken verstanden werden, mehr vorliegende Daten führen allerdings nicht per se zu besseren und mehr Therapieangeboten. In vielerlei Hinsicht muss gerade ein breites Forschungsverständnis dafür werben, dass Forschung auch in Sackgassen und nicht-anwendungsfähige Erkenntnisse führen kann. Somit ist dann zu fragen, ob es sich nicht vielmehr um Solidarität mit Forschenden und deren Erkenntnisinteresse handelt - dies könnte jedoch den Solidaritätsbegriff überstrapazieren. Partizipative Strukturen, die sowohl transparent als auch deliberativ solche Zwecke und Ziele von Forschung offenlegen und hierfür Unterstützung erhalten, wecken dann keine falschen Hoffnungen, die ja in der Forderung nach Solidarität ebenfalls beinhaltet sind - nämlich der Nutzen für andere. Des Weiteren bleibt wichtig zu reflektieren, mit welchen Individuen und Gruppenmitgliedern kollektive Solidarität empfunden wird - und wie sich dieser Kreis an Kollektiven sowohl in Raum (global) oder in Zeit (spätere Generationen) und über die eigene Betroffenheit hinaus ausbreiten kann.

Der vorliegende Beitrag argumentiert vor diesem Hintergrund dafür, dass die drei Konzepte Kollektivität, Partizipation und Solidarität für eine ethische Bewertung neuer Technologien und Innovationen in der Biomedizin in unserer Gesellschaft unerlässlich sind und etablierte Prinzipien wie Selbstbestimmung, Nicht-Schaden, Fürsorge und Gerechtigkeit sinnvoll ergänzen. Die komplementäre, kollektiv-orientierte Betrachtung legt den Fokus vermehrt auf das Individuum - hier als Bürger*innen, Patient*innen und Betroffene - in seinem jeweiligen Verhältnis zu sozialen Gruppen und Kollektiven. Partizipation und Solidarität sind dabei zwei exemplarische Konzepte. In der aktuellen pandemischen Situation, in der es zu weitreichenden Public-Health-Maßnahmen gekommen ist, sind Fragen, die das Verhältnis von Individuum und Kollektiv betreffen, besonders sinnfällig geworden: Individuelle Rechte und Grundwerte auf der einen Seite und epidemiologische Forschung sowie behördliche Schutzmaßnahmen im Namen „der Bevölkerung“ oder vulnerablen Bevölkerungsgruppen auf der anderen Seite treten in eine normative Spannung (siehe Fangerau/Labisch, Kap. 20). Die Frage, ob Individuen legitimerweise verpflichtet oder nur motiviert werden dürfen, sich solidarisch zu verhalten, wird besonders dann kontrovers diskutiert, wenn man deutlich macht, dass ggf. nicht nur vulnerable Gruppen im eigenen Land im Fokus stehen 
sollten, sondern auch die gesamte europäische oder gar globale Bevölkerung. Während allgemeine Solidaritätsaufforderungen sicher unproblematisch sind, ist noch viel Reflexionsarbeit in der Beantwortung der legitimen sozialen Reichweite und zumutbaren sozialen Kosten für den jeweiligen Fall zu leisten. Es ist notwendig, sich diesen Details sowohl in ihrer Verbindlichkeit als auch ihrer Reichweite ausführlicher zu widmen. Auch erscheinen Aufrufe zu Solidarität und Partizipation an Gesundheitsmaßnahmen nicht immer transparent kommuniziert: Was ist das Kollektiv, zu dem sich solidarisch verhalten werden soll? Welchen Kollektiven soll die datenintensive Forschung, zu dessen Teilnahme gebeten wird, Nutzen bringen? In welchem Verhältnis stehen schließlich Patient*innen und Proband*innen zu gesundheitsbezogenen Kollektiven wie Patient*innenorganisationen, Gesundheitsverbänden, gesundheitsökonomischen und staatlichen Organisationen in der gesundheitspolitischen Gestaltung?

Die bioethische Diskussion hat mit den ersten Untersuchungen und konzeptuellen Vorschlägen zu Partizipation, Solidarität und Kollektivität bereits einiges an Vorarbeit geleistet. Angesichts der digitaltechnologischen Entwicklungen und der pandemischen Situation erscheint ihre Vertiefung dringend notwendig. Deren Diskussion und Elaboration sollte nun am besten interdisziplinär, betroffenenorientiert und, ja, in kollektiver Analyse und Reflexion erfolgen.

\subsection{Literaturverzeichnis}

Allmendinger, J. (2020): Zurück in alte Rollen. Corona bedroht die Geschlechtergerechtigkeit. In: WZB Mitteilungen 168: 45-47.

Beier, K. et al. (2016): Understanding collective agency in bioethics. In: Med Health Care Philos 19(3): 411-422.

Beier, K. et al. (2019): Taking patient involvement seriously. A critical ethical analysis of participatory approaches in data-intensive medical research. In: BMC Med Inform Decis Mak, Online-Publikation 25.04.2016. DOI: 10.1186/s12911-019-0799-7.

Blasimme, A./Vayena, E. (2016): Becoming partners, retaining autonomy. Ethical considerations on the development of precision medicine. In: BMC Med Ethics, Online-Publikation 04.11.2016. DOI: 10.1186/s12910-016-0149-6.

Blasimme A./ Vayena, E (2020): What's next for COVID-19 apps? Governance and oversight. In: Science 370: 760-762.

Bratman, M. E. (1993): Shared intention. In: Ethics 104(1): 97-113.

Bratman, M. E. (2006): Dynamics of sociality. In: French, P. A./Wettstein, H. K. (Hrsg.): Shared intentions and collective responsibility. Blackwell, Boston/Oxford: 1-15.

Jennings, B. (2018): Solidarity and care as relational practices. In: Bioethics 32(9): 553-561. 
Johnson, J. L. et al. (2004): Othering and being othered in the context of health care services. In: Health Commun 16(2): 255-271.

Jongsma, K. et al. (2018): One for all, all for one? Collective representation in healthcare policy. In: JBI 15(3): 337-340.

Kaye, J. et al. (2012): From patients to partners. Participant-centric initiatives in biomedical research. In: Nat Rev Genet 13(5): 371-376.

Kelty, C. et al. (2015): Seven dimensions of contemporary participation disentangled. In: J Assoc Inf Sci Technol 66(3): 474-488.

Kolers, A. (2021): What does solidarity do for bioethics? In: J Med Ethics 47(2): 122-128.

Kymlicka, W. (1991): Liberalism, community, and culture. Clarendon Press, Oxford/New York.

Marschelke, J. C. (2019): Doing collectivity. Eine praxeologische Annäherung an Kollektivität. In: Zeitschrift für Kultur- und Kollektivwissenschaft 5(1): 79-114.

McKenna, M. (2006): Collective responsibility and agent meaning theory. In: Midwest Stud Philos 30(1): 16-34.

Mittelstadt, B. D./Floridi, L. (2016): The ethics of big data. Current and foreseeable issues in biomedical contexts. In: dies. (Hrsg.): The ethics of biomedical big data. Springer, Cham: 445-480.

Neuhaus, C. P. (2020): Does solidarity require „all of us” to participate in genomics research? In: Hastings Center Report 50(1): 62-69.

Prainsack, B. (2017a): Personalized medicine. Empowered patients in the $21^{\text {st }}$ century? New York University Press, New York.

Prainsack, B. (2017b): Research for personalised medicine. Time for solidarity. In: Med Law 36(1): 87-98.

Prainsack, B./Buyx, A. (2011): Solidarity. Reflections on an emerging concept in bioethics. Nuffield Council on Bioethics, London.

Prainsack, B./Buyx, A. (2016): Das Solidaritätsprinzip. Ein Plädoyer für eine Renaissance in Medizin und Bioethik. Campus Verlag, Frankfurt am Main.

Rehbein, B. (2015): Das Kollektiv in der Soziologie: Gesellschaft, Klasse und Milieu. In: Zeitschrift für Kultur- und Kollektivwissenschaft 1(1): 11-28.

Samerski, S./Müller, H. (2019): Digital health literacy. Thesen zu Konzept und Förderungsmöglichkeiten. In: Pfannstiel, M. et al. (Hrsg.): Digitale Transformation von Dienstleistungen im Gesundheitswesen VI. Springer Gabler, Wiesbaden.

Schicktanz, S. (in Vorbereitung): Collectivity in bioethics.

Schicktanz S. et al. (2012): The ethics of „public understanding of ethics”-why and how bioethics expertise should include public and patients' voices. In: Med Health Care and Philos 15: 129-139.

Searle, J. R. (1990): Collective intentions and actions. In: Cohen, P. R. et al. (Hrsg.): Intentions in communication. MIT Press, Cambridge: 401-415.

Searle, J. R. (2010): Making the social world. The structure of human civilization. Oxford University Press, New York. 
Spivak, G. C. (1985): The Rani of Sirmur. An essay in reading the archives. In: Hist Theo 24(3): 247-272. Stjernø, S. (2009): Solidarity in Europe. The history of an idea. Cambridge University Press, Cambridge/New York.

Tagesschau (2020): Steinmeier zur Corona-Krise: „Solidarität ist jetzt existenziell wichtig“. 26.03.2020. Unter: https://www.tagesschau.de/inland/steinmeier-coronavirus-103.html [22.02.2021].

Tagesschau (2021): Impfstoff-Verteilung: „Eine Frage der Menschlichkeit“. 22.01.2021. Unter: https:// www.tagesschau.de/ausland/corona-impfstoff-steinmeier-who-101.html [22.02.2021].

Taylor, C. (1995): Philosophical arguments. 2. Auflage. Havard University Press, Cambridge/ London.

Ter Meulen, R. H. J. et al. (Hrsg.) (2001): Solidarity in health and social care in Europe. Kluwer Academic Publishers, Dodrecht/Boston/London.

Wachtler, B./Hoebel, J. (2020): Soziale Ungleichheit und COVID-19. Sozialepidemiologische Perspektiven auf die Pandemie. In: Das Gesundheitswesen 82(08/09): 670-675.

Warsh, J. (2014): PPI: Understanding the difference between patient and public involvement. In: The American Journal of Bioethics 14(6): 25-26.

Wicks, P. et al. (2011): Accelerated clinical discovery using self-reported patient data collected online and a patient-matching algorithm. In: Nature Biotechnology 29: 411-414.

Williams, M. S. (1998): Voice, trust and memory. Marginalized groups and the failings of liberal representation. Princeton University Press, Princeton.

Wöhlke S. et al. (2018): Physicians' communication patterns for motivating rectal cancer patients to biomarker research: Empirical insights and ethical issues. In: Clinical Ethics 13: 175-188.

Woods, S. (2016): Big data governance. Solidarity and the patient voice. In: Mittelstadt, B. D./Floridi, L. (Hrsg.): The ethics of biomedical big data. Springer, Cham: 221-238.

Woolley, J. P. et al. (2016): Citizen science or scientific citizenship? Disentangling the uses of public engagement rhetoric in national research initiatives. In: BMC Med Ethics, Online-Publikation 04.06.2016. DOI: 10.1186/s12910-016-0117-1.

Xu, J. et al. (2019): Translating cancer genomics into precision medicine with artificial intelligence. Applications, challenges and future perspectives. In: Hum Genet 138(2): 109-124.

Young, I. M. (1997): Intersecting voices. Dilemmas of gender, political philosophy, and policy. Princeton University Press, Princeton. 